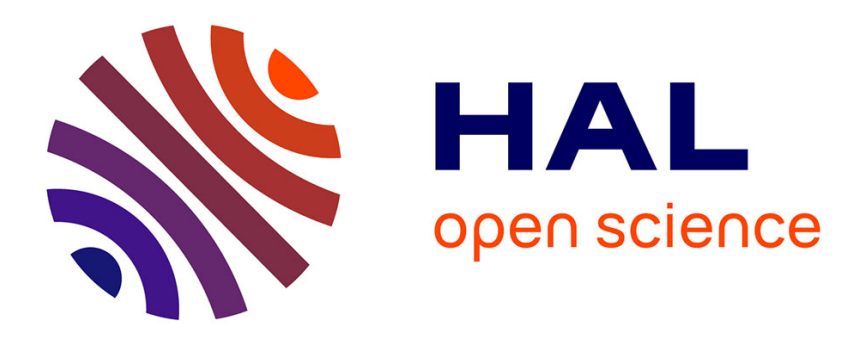

\title{
An exactly soluble non-equilibrium system: The asymmetric simple exclusion process
}

\author{
Bernard Derrida
}

\section{To cite this version:}

Bernard Derrida. An exactly soluble non-equilibrium system: The asymmetric simple exclusion process. Physics Reports, 1998, 301 (1), pp.65-83. 10.1016/S0370-1573(98)00006-4 . hal-03282986

\section{HAL Id: hal-03282986 \\ https://hal.science/hal-03282986}

Submitted on 19 Jul 2021

HAL is a multi-disciplinary open access archive for the deposit and dissemination of scientific research documents, whether they are published or not. The documents may come from teaching and research institutions in France or abroad, or from public or private research centers.
L'archive ouverte pluridisciplinaire HAL, est destinée au dépôt et à la diffusion de documents scientifiques de niveau recherche, publiés ou non, émanant des établissements d'enseignement et de recherche français ou étrangers, des laboratoires publics ou privés. 


\title{
An exactly soluble non-equilibrium system: The asymmetric simple exclusion process
}

\author{
B. Derrida*
}

Laboratoire de Physique Statistique, Ecole Normale Supérieure, 24 rue Lhomond, 75005 Paris, France

\begin{abstract}
A number of exact results have been obtained recently for the one-dimensional asymmetric simple exclusion process, a model of particles which hop to their right at random times, on a one-dimensional lattice, provided that the target site is empty. Using either a matrix form for the steady-state weights or the Bethe ansatz, several steady-state properties can be calculated exactly: the current, the density profile for open boundary conditions, the diffusion constant of a tagged particle. The matrix form of the steady state can be extended to calculate exactly the steady state of systems of two species of particles and shock profiles. (C) 1998 Elsevier Science B.V. All rights reserved.
\end{abstract}

PACS: $05.40 .+\mathrm{j} ; 05.60 .+\mathrm{w}$

\section{Introduction}

In equilibrium statistical mechanics, one usually associates an energy function $E(C)$ to every possible configuration $C$ of a system. At equilibrium, each configuration $C$ has a weight proportional to $\exp [-E(C) / T]$

$$
P_{\mathrm{eq}}(C)=Z^{-1} \mathrm{e}^{-E(C) / T},
$$

where $T$ is the temperature and $Z$ the partition function. The problem is then to calculate equilibrium properties by averaging over all configurations weighted by Eq. (1).

The dynamics towards such an equilibrium is often described by a master equation of the form

$$
\frac{\mathrm{d} P(C)}{\mathrm{d} t}=\sum_{C^{\prime}} M\left(C, C^{\prime}\right) P\left(C^{\prime}\right),
$$

\footnotetext{
*E-mail: bernard.derrida@physique.ens.fr.
} 
where $M\left(C, C^{\prime}\right) \mathrm{d} t$ is the probability of a transition from configuration $C^{\prime}$ to configuration $C$ during the infinitesimal time interval $\mathrm{d} t$. To conserve the probability, the diagonal term has to satisfy

$$
M(C, C)=-\sum_{C^{\prime} \neq C} M\left(C^{\prime}, C\right) .
$$

( $-M(C, C) P(C) \mathrm{d} t$ represents the probability of escaping from configuration $C$ during the time interval $\mathrm{d} t$ ). To insure that a distribution $P(C)$ evolving according to Eq. (2) has a long time limit independent of the initial condition, the rule of evolution $M\left(C, C^{\prime}\right)$ should not leave any subset of configurations disconnected from the rest (i.e. there should be a path of non-zero matrix elements connecting any pair of configurations). Moreover, the simplest way for the limit to be $P_{\text {eq }}(C)$ given by Eq. (1) is to choose a matrix $M\left(C, C^{\prime}\right)$ which satisfies detailed balance, meaning that for any pair of configurations $C_{1}, C_{2}$

$$
M\left(C_{1}, C_{2}\right) P_{\text {eq }}\left(C_{2}\right)=M\left(C_{2}, C_{1}\right) P_{\text {eq }}\left(C_{1}\right) .
$$

In out-of-equilibrium systems, there is usually no energy function $E(C)$ and the system is only defined by its dynamical rules. Typically, for a system with stochastic dynamics, only a matrix $M$ is given, and the evolution of the weights $P(C)$ is governed by the master equation (2). In the long-time limit, the system usually (if all the configurations are connected) reaches a steady state $P_{\text {steady state }}(C)$ which in general does not satisfy the detailed balance condition (3) but only the weaker condition of stationarity:

$$
\sum_{C^{\prime}} M\left(C^{\prime}, C\right) P_{\text {steady state }}(C)=\sum_{C^{\prime}} M\left(C, C^{\prime}\right) P_{\text {steady state }}\left(C^{\prime}\right) .
$$

Eq. (4) expresses the fact that the probabilities of entering and of leaving the configuration $C$ during the time interval $\mathrm{d} t$ are equal. (The stationarity conditions (4) are weaker than detailed balance (3) since for a system of $\Omega$ configurations, stationarity imposes $\Omega$ equations (4) whereas detailed balance (3) usually requires many more conditions.)

A trivial example of system reaching a steady state without satisfying detailed balance (3) is the problem of a biased random walker on a ring of $N$ sites. During each infinitesimal time interval $\mathrm{d} t$, the walker hops with probability $p \mathrm{~d} t$ to its right and $q \mathrm{~d} t$ to its left. There are $N$ possible positions $i$ for the walker and the solution of Eq. (4) for the steady state probability is just $P_{\text {steady state }}(i)=1 / N$. Obviously, $M(i+1, i) P_{\text {steady state }}(i)=p / N \neq q / N=M(i, i+1) P_{\text {steady state }}(i+1)$ and detailed balance (3) is not satisfied as soon as there is a bias (i.e. $p \neq q$ ).

In general, the steady state weights $P_{\text {steady state }}(C)$ have to be determined by solving the equations (Eq. (4)) and the expressions obtained are usually complicated. Once the steady state weights are known, the calculation of steady state properties becomes very similar to that of equilibrium properties: one has to average physical quantities with the steady state weights.

One of the simplest non-equilibrium systems one can consider is the fully asymmetric simple exclusion process ASEP in one dimension [1-4]. The model describes a driven lattice gas [5] in one dimension with hard core repulsion. At large scale [6], the ASEP is expected [7-10] to be described by a noisy Burgers equation or equivalently by the Kardar-Parisi-Zhang equation. Therefore, the long time and large scale properties of the ASEP can be reinterpreted as asymptotic properties of directed polymers in a random medium or of growing interfaces. 
In one dimension, the ASEP can be defined as follows. Each site of a one-dimensional lattice is either occupied by one particle or empty. A configuration of the system can therefore be characterized by binary variables $\left\{\tau_{i}\right\}$ where $\tau_{i}=1$ if site $i$ is occupied by a particle and $\tau_{i}=0$ if site $i$ is empty. During every infinitesimal time interval $\mathrm{d} t$, each particle hops with probability $\mathrm{d} t$ to its right if this site is empty (and does not move otherwise).

For any initial configuration $\left\{\tau_{i}(0)\right\}$, one can write the evolution of arbitrary correlation functions by considering the possible events which occur during the time interval $\mathrm{d} t$. For example, the evolution of the occupation $\tau_{i}$ of site $i$ during the time interval $\mathrm{d} t$ is given by

$$
\tau_{i}(t+\mathrm{d} t)= \begin{cases}\tau_{i}(t) & \text { with probability } 1-2 \mathrm{~d} t \\ \tau_{i-1}(t)+\tau_{i}(t)-\tau_{i-1}(t) \tau_{i}(t) & \text { with probability } \mathrm{d} t, \\ \tau_{i}(t) \tau_{i+1}(t) & \text { with probability } \mathrm{d} t,\end{cases}
$$

(the first line corresponds to updating neither the bond $(i-1, i)$ nor the bond $(i, i+1)$, the second line corresponds to updating the bond $(i-1, i)$ and the third line corresponds to updating the bond $(i, i+1))$ and the average over the history between times 0 and $t$ leads to

$$
\frac{\mathrm{d}\left\langle\tau_{i}\right\rangle}{\mathrm{d} t}=\left\langle\tau_{i-1}\right\rangle-\left\langle\tau_{i}\right\rangle-\left\langle\tau_{i-1} \tau_{i}\right\rangle+\left\langle\tau_{i} \tau_{i+1}\right\rangle .
$$

For a pair of neighboring sites, one can write

$$
\begin{aligned}
\tau_{i}(t & +\mathrm{d} t) \tau_{i+1}(t+\mathrm{d} t) \\
& =\left\{\begin{array}{l}
\tau_{i}(t) \tau_{i+1}(t) \\
\left(\tau_{i-1}(t)+\tau_{i}(t)-\tau_{i-1}(t) \tau_{i}(t)\right) \tau_{i+1}(t) \\
\tau_{i}(t) \tau_{i+1}(t) \\
\tau_{i}(t) \tau_{i+1}(t) \tau_{i+2}(t)
\end{array}\right.
\end{aligned}
$$

with probability $1-3 \mathrm{~d} t$, with probability $\mathrm{d} t$, with probability $\mathrm{d} t$, with probability $\mathrm{d} t$,

which gives for the evolution equation of $\left\langle\tau_{i} \tau_{i+1}\right\rangle$

$$
\frac{\mathrm{d}\left\langle\tau_{i} \tau_{i+1}\right\rangle}{\mathrm{d} t}=\left\langle\tau_{i-1} \tau_{i+1}\right\rangle-\left\langle\tau_{i} \tau_{i+1}\right\rangle-\left\langle\tau_{i-1} \tau_{i} \tau_{i+1}\right\rangle+\left\langle\tau_{i} \tau_{i+1} \tau_{i+2}\right\rangle
$$

and a similar reasoning allows one to derive the evolution equations of higher correlations. What is visible in Eqs. (6) and (7) is that all the correlation functions are coupled in the hierarchy. To determine in Eq. (6) the one-point functions $\left\langle\tau_{i}\right\rangle$, one needs to know the two-point functions $\left\langle\tau_{i} \tau_{i+1}\right\rangle$. Trying to calculate these nearest-neighbor correlations in Eq. (7) requires the knowledge of further correlations $\left\langle\tau_{i-1} \tau_{i+1}\right\rangle$ or of higher-order correlations $\left\langle\tau_{i-1} \tau_{i} \tau_{i+1}\right\rangle$ and so on. This shows that the problem is a $N$-body problem and so far it has not been possible to derive an expression of the correlations at time $t$, given an arbitrary initial condition $\left\{\tau_{i}(0)\right\}$.

\section{The steady state for periodic conditions}

For periodic boundary conditions, its turns out that the steady state of the ASEP is particularly simple. For a system of $P$ particles on a ring of $N$ sites, one can show that in the steady state all 
possible configurations have equal weight $[11]$ as

$$
P_{\text {steady state }}(C)=\frac{P !(N-P) !}{N !} .
$$

This is because, if $n(C)$ is the number of clusters in the configuration $C$, the probability of leaving configuration $C$ during the time interval $\mathrm{d} t$ is $n(C) P(C) \mathrm{d} t$ (by the move of the first particle of each cluster) whereas the probability of entering the configuration $C$ is $\sum_{C^{\prime}} P\left(C^{\prime}\right) \mathrm{d} t$ where the sum runs over the configurations $C^{\prime}$ obtained from $C$ by moving one step backwards of the last particle of each cluster. As the number of configurations $C^{\prime}$ is equal to the number of clusters $n(C)$, the stationarity condition (4) is fulfilled when all configurations have equal weight.

This simplicity of the steady state makes the calculation of equal time correlation functions very easy. For example

$$
\left\langle\tau_{i}\right\rangle=\frac{P}{N} ; \quad\left\langle\tau_{i} \tau_{j}\right\rangle=\frac{P(P-1)}{N(N-1)} ; \quad\left\langle\tau_{i} \tau_{j} \tau_{k}\right\rangle=\frac{P(P-1)(P-2)}{N(N-1)(N-2)} .
$$

The fact that the weights (8) of the configurations in the steady state are simple, Eq. (8) does not make all the steady state properties easy to calculate. For example, unequal time correlation functions like $\left\langle\tau_{i}(0) \tau_{j}(t)\right\rangle$ are not known, even in the steady state. One of the simplest quantity which contains some information about unequal time correlations is the diffusion constant $\Delta$ of a tagged particle [12-14] on the ring. If we tag one of the particles (without in anyway changing its dynamics) and call $Y_{t}$ the number of hops the particle has made up to time $t$, one expects that the following two limits exist:

$$
\lim _{t \rightarrow \infty} \frac{\left\langle Y_{t}\right\rangle}{t}=v ; \quad \lim _{t \rightarrow \infty} \frac{\left\langle Y_{t}^{2}\right\rangle-\left\langle Y_{t}\right\rangle^{2}}{t}=\Delta
$$

and these two limits define the velocity $v$ and the diffusion constant $\Delta$. The exact expressions of $v$ and of $\Delta$ are given by [15]

$$
\begin{aligned}
& v=\frac{N-P}{N-1}, \\
& \Delta=\frac{(2 N-3) !}{(2 P-1) !(2 N-2 P-1) !}\left[\frac{(P-1) !(N-P) !}{(N-1) !}\right]^{2} .
\end{aligned}
$$

The expression for $v$ is a simple consequence of the fact (8) that all configurations with $P$ particles have equal probability [11] in the steady state. The expression for $\Delta$, on the other hand, is harder to obtain and was first derived in [15] using a generalization of the matrix approach discussed in Section 3. We will see in Section 4 that it can also be recovered using the Bethe ansatz.

The expression of $\Delta$ in terms of correlation functions is usually complicated. However, for the ASEP on a ring with periodic boundary conditions, using the fact (8) that all configurations have equal weight, one can show that

$$
\Delta=\frac{N(N-P)}{P(N-1)}+\frac{2 N^{2}}{P^{2}} \int_{0}^{\infty} \mathrm{d} t\left\{\left\langle\tau_{i}(t)\left[1-\tau_{i+1}(t)\right]\left[1-\tau_{i}(0)\right] \tau_{i+1}(0)\right\rangle-\frac{P^{2}(N-P)^{2}}{N^{2}(N-1)^{2}}\right\} .
$$


For large $N$, keeping the density $\rho=P / N$ of particles fixed, Eq. (11) becomes

$$
\Delta \simeq \frac{\sqrt{\pi}}{2}\left[\frac{(1-\rho)^{3 / 2}}{\rho^{1 / 2}}\right] \frac{1}{N^{1 / 2}} .
$$

The fact that $\Delta$ vanishes for $N \rightarrow \infty$ indicates that in the infinite system, for fixed initial conditions, the fluctuations in the distance travelled by a tagged particle are subdiffusive [12-14].

\section{The steady state for open boundary conditions}

Another case for which the weights of the configurations in the steady state can be calculated exactly $[16,17]$ is the ASEP with open boundary conditions [4] which is defined as follows: each site $i$ of a one-dimensional lattice of $N$ sites is either occupied $\left(\tau_{i}=1\right)$ by a particle or empty $\left(\tau_{i}=0\right)$; during a time interval $\mathrm{d} t$ (with $\mathrm{d} t \ll 1$ ), each particle has a probability $\mathrm{d} t$ of hopping to its right, provided the target site is empty; moreover, during the time interval $\mathrm{d} t$, a particle may enter the lattice at site 1 with probability $\alpha \mathrm{d} t$ (if this site is empty) and a particle at site $N$ may leave the lattice with probability $\beta \mathrm{d} t$ (if this site is occupied).

The evolution equations (Eqs. (6) and (7)) remain valid in the bulk (i.e., as long as all the sites involved in the equations belong to the system). At the boundary, they are modified to include the boundary effects. For example Eq. (6) becomes

$$
\begin{aligned}
& \frac{\mathrm{d}\left\langle\tau_{1}\right\rangle}{\mathrm{d} t}=\alpha\left\langle\left(1-\tau_{1}\right)\right\rangle-\left\langle\tau_{1}\left(1-\tau_{2}\right)\right\rangle, \\
& \frac{\mathrm{d}\left\langle\tau_{N}\right\rangle}{\mathrm{d} t}=\left\langle\tau_{N-1}\left(1-\tau_{N}\right)\right\rangle-\beta\left\langle\tau_{N}\right\rangle .
\end{aligned}
$$

In the steady state, all the equal time correlation functions become time independent. In particular, the left-hand sides of Eqs. (6), (7), (14) and (15) vanish.

The steady state properties of this asymmetric exclusion model with open boundary conditions can be calculated exactly [16-18] for any $N, \alpha$ and $\beta$. The solution described below consists in writing the steady state weights of the configurations as the matrix elements of products of $N$ matrices [17]. This type of approach, initially introduced in connection with the Bethe ansatz $[19,20]$, has been used to solve several problems in statistical mechanics, in particular, the problem of directed lattice animals [21].

The main idea is to write the weight $P_{\text {steady state }}\left(\tau_{1} \cdots \tau_{N}\right)$ of each configuration in the steady state as

$$
P_{\text {steady state }}\left(\tau_{1} \cdots \tau_{N}\right)=Z^{-1}\left\langle W\left|\prod_{i=1}^{N}\left[\tau_{i} D+\left(1-\tau_{i}\right) E\right]\right| V\right\rangle,
$$

where $D, E$ are matrices, $\langle W|| V$,$\rangle are vectors, the \tau_{i}$ are the occupation variables and the normalization factor $Z$ is given by

$$
Z=\sum_{\tau_{1}=1,0} \ldots \sum_{\tau_{N}=1,0}\left\langle W\left|\prod_{i=1}^{N}\left[\tau_{i} D+\left(1-\tau_{i}\right) E\right]\right| V\right\rangle=\left\langle W\left|(D+E)^{N}\right| V\right\rangle .
$$


In other words, in the product (16) a matrix $D$ appears whenever a site is occupied $\left(\tau_{i}=1\right)$ and $E$ whenever a site is empty $\left(\tau_{i}=0\right)$. Since $D$ and $E$ do not commute in general, the weights $P_{\text {steady state }}\left(\tau_{1} \cdots \tau_{N}\right)$ are complicated functions of the configuration $\left\{\tau_{1} \cdots \tau_{N}\right\}$.

In [17] it was shown that Eqs. (16) and (17) do give the weights in the steady state of the asymmetric exclusion model when the matrices $D$ and $E$ and the vectors $\langle W|$ and $|V\rangle$ satisfy the following algebraic rules:

$$
\begin{aligned}
& D E=D+E, \\
& \langle W| E=\frac{1}{\alpha}\langle W|, \\
& D|V\rangle=\frac{1}{\beta}|V\rangle .
\end{aligned}
$$

Proofs that this is so are rather easy and have already been published [17,22]. Let us just explain it with a simple example. Consider a configuration of the form

$$
\underbrace{00 \cdots 0}_{p} \underbrace{1 \cdots 1}_{N-p} .
$$

During each time interval $\mathrm{d} t$, the probability of entering this configuration is

$$
\frac{\left\langle W\left|E^{p-1} D E D^{N-p-1}\right| V\right\rangle}{\left\langle W\left|(D+E)^{N}\right| V\right\rangle} \mathrm{d} t
$$

by coming from configuration

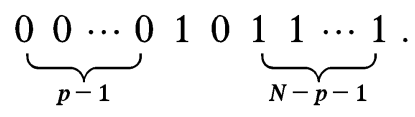

The probability of leaving this configuration is given by

$$
(\alpha+\beta) \frac{\left\langle W\left|E^{p} D^{N-p}\right| V\right\rangle}{\left\langle W\left|(D+E)^{N}\right| V\right\rangle} \mathrm{d} t
$$

since one particle may enter at site 1 or the particle at site $N$ may leave. In the steady state these two expressions should be equal (4) to ensure that the weight of configuration (21) remains unchanged

$$
\left\langle W\left|E^{p-1} D E D^{N-p-1}\right| V\right\rangle=(\alpha+\beta)\left\langle W\left|E^{p} D^{N-p}\right| V\right\rangle
$$

and this equality is easy to check using Eqs. (18)-(20). A generalization of this reasoning to arbitrary configurations is given in [22] and provides a proof that Eq. (16) gives the steady state weight of all the configurations. 
Once the weights are known from Eqs. (16) and (17), one can calculate all the equal time steady state properties. For example, the average occupation $\left\langle\tau_{i}\right\rangle$ of site $i$ is given by

$$
\left\langle\tau_{i}\right\rangle=\sum_{\tau_{1}=0,1} \cdots \sum_{\tau_{N}=0,1} \tau_{i} P_{\text {steady state }}\left(\tau_{1} \cdots \tau_{N}\right),
$$

which becomes in terms of the matrices $D$ and $E$ and the vectors $\langle W|$ and $|V\rangle$

$$
\left\langle\tau_{i}\right\rangle=\frac{\left\langle W\left|(D+E)^{i-1} D(D+E)^{N-i}\right| V\right\rangle}{\left\langle W\left|(D+E)^{N}\right| V\right\rangle} .
$$

We see that, to evaluate Eq. (27), one has to calculate the matrix elements of the form

$$
\left\langle W\left|D^{m_{1}} E^{n_{1}} D^{m_{2}} E^{n_{2}} \ldots\right| V\right\rangle .
$$

One way to do it $[17,22]$ is to construct matrices and vectors which satisfy Eqs. (18)-(20). One can show, however, that the calculation of all these matrix elements can be done directly from the algebraic rules Eqs. (18)-(20) without using any specific representation of the algebra. First, from Eqs. (19) and (20), it is obvious that all the matrix elements of the form $\left\langle W\left|E^{p} D^{q}\right| V\right\rangle$ are easy to calculate and is given by

$$
\left\langle W\left|E^{p} D^{q}\right| V\right\rangle=\frac{1}{\alpha^{p} \beta^{q}}\langle W \mid V\rangle .
$$

It is also easy to see, using $D E=D+E$, that any more complicated matrix element (28) can be reduced to simple matrix elements of the form (29). For example, the matrix element $\left\langle E^{3} D E^{2} D\right\rangle$ becomes, using Eq. (18)

$$
\left\langle E^{3} D E^{2} D\right\rangle=\left\langle E^{5} D\right\rangle+\left\langle E^{4} D\right\rangle+\left\langle E^{3} D^{2}\right\rangle .
$$

In particular, one can obtain that way the expression of the denominator $\left\langle W\left|(D+E)^{N}\right| V\right\rangle$ for all $N$ as

$$
\frac{\left\langle W\left|(D+E)^{N}\right| V\right\rangle}{\langle W \mid V\rangle}=\sum_{p=1}^{N} \frac{p(2 N-1-p) !}{N !(N-p) !} \frac{\beta^{-p-1}-\alpha^{-p-1}}{\beta^{-1}-\alpha^{-1}} .
$$

In the large $N$ limit, one can calculate from Eq. (27) the average occupation $\rho=\left\langle\tau_{i}\right\rangle$ for a site $i$ far from the boundaries and one finds the phase diagram shown in Fig. 1 with the three following phases:

Phase I: For $\alpha>\frac{1}{2}$ and $\beta>\frac{1}{2}$,

$$
\rho=\frac{1}{2} \text {. }
$$

Phase II: For $\alpha<\frac{1}{2}$ and $\alpha<\beta$,

$$
\rho=\alpha .
$$

Phase III: For $\beta<\frac{1}{2}$ and $\beta<\alpha$,

$$
\rho=1-\beta .
$$



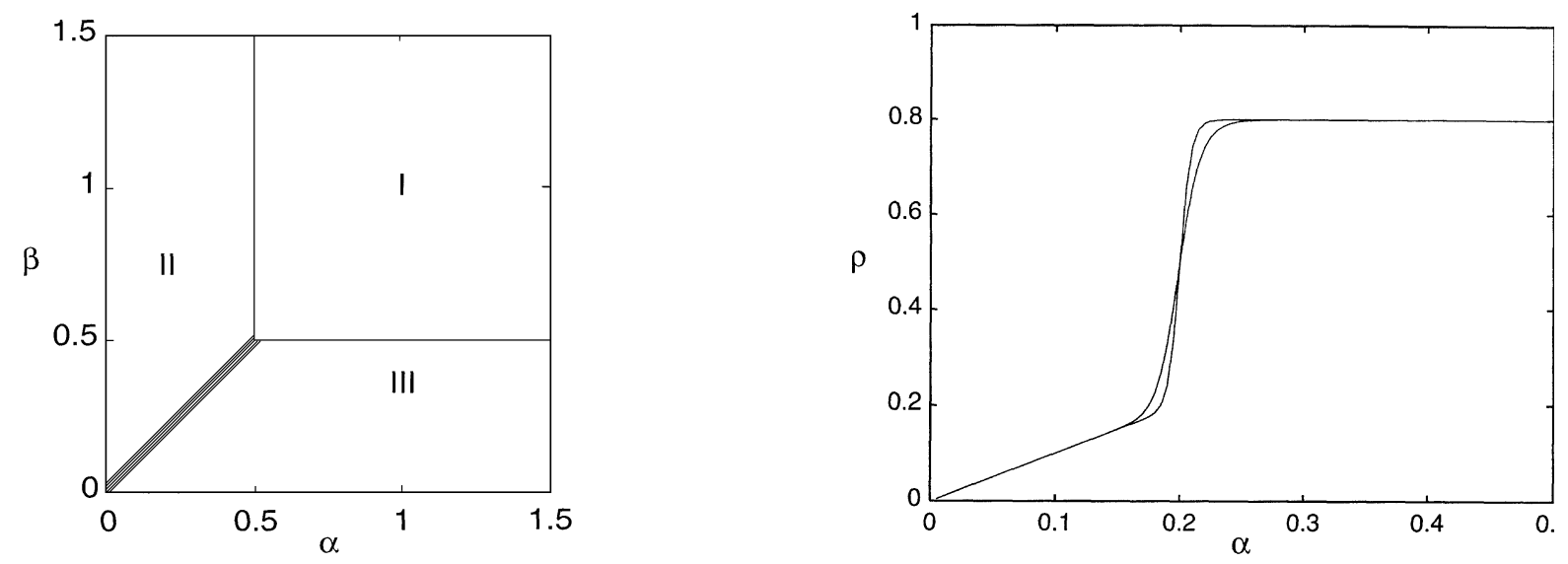

Fig. 1. The phase diagram.

Fig. 2. The average occupation $\rho=\left\langle\tau_{(N+1) / 2}\right\rangle$ of the central site versus $\alpha$ for $N=61$ and $N=121$ when $\beta=0.2$.

Phase I is the maximal current phase, phase II is a low density phase and phase III is a high density phase. The lines $\alpha=\frac{1}{2}, \beta>\frac{1}{2}$ and $\beta=\frac{1}{2}, \alpha>\frac{1}{2}$ are second-order transition lines ( $\rho$ is continuous) whereas the line $\alpha=\beta<\frac{1}{2}$ is a first-order transition line ( $\rho$ is discontinuous). The first-order transition can be seen in Fig. 2 where the average occupation of the central site $\rho=\left\langle\tau_{(N+1) / 2}\right\rangle$ is plotted versus $\alpha$ (at $\beta=0.2$ fixed) for two system sizes $N=61$ and $N=121$. As $N$ increases, one observes that $\rho$ becomes discontinuous along the first-order transition line $\alpha=\beta$.

There is a line in the phase diagram, the line

$$
\alpha+\beta=1
$$

along which there exists a solution of Eqs. (18)-(20) such that the matrices $D$ and $E$ commute. If one chooses $D$ and $E$ to be numbers, one must have from Eqs. (19) and (20), $D=1 / \beta$ and $E=1 / \alpha$ and condition (18) imposes that $1 / \alpha+1 / \beta=1 /(\alpha \beta)$ which leads to Eq. (34). Along this line, there is no correlation between the different sites and

$$
P_{\text {steady state }}\left(\tau_{1} \cdots \tau_{N}\right)=\prod_{i=1}^{N}\left[\tau_{i} \alpha+\left(1-\tau_{i}\right)(1-\alpha)\right] .
$$

This line plays the role of a disordered line in usual statistical mechanics models [23].

One can try to calculate for the ASEP with open boundary conditions unequal time correlations. Because the steady state is more complicated than in the case of periodic boundary conditions, unequal time correlations are also much harder to obtain. So far only the diffusion constant of the integrated current, which generalises expression (11) to the case of open boundary conditions, has been calculated exactly [24]. However, contrarily to what was said in [24], the relation to unequal time correlation functions is usually much more complicated than (12). 


\section{The Bethe ansatz method}

For the ASEP, the Bethe ansatz was first used [25-27] to calculate the gap which gives the largest relaxation time in the system. In this section we will see that it gives also the diffusion constant (11) in the case of periodic boundary conditions [28].

Because on a ring the particles cannot overtake each other, they all cover the same distance $Y_{t}$, up to fluctuations which remain bounded when $t$ becomes large. Therefore, if one calls $X_{t}$ the total distance covered by the three particles during the time $t$, one has $X_{t} \simeq P Y_{t}$ or more precisely, if one considers the generating function of $X_{t}$ and of $Y_{t}$, the two following limits coincide

$$
\lim _{t \rightarrow \infty} \frac{\log \left\langle\mathrm{e}^{\gamma X_{t}}\right\rangle}{t}=\lim _{t \rightarrow \infty} \frac{\log \left\langle\mathrm{e}^{P \gamma Y_{t}}\right\rangle}{t}=\lambda(\gamma)
$$

and their common value $\lambda(\gamma)$ can be calculated using the Bethe ansatz.

To see how the Bethe ansatz works, it is easier to consider a system of three particles $(P=3)$ on a ring of $N$ sites. One can show [28] that $\lambda(\gamma)$ is the largest eigenvalue of a $N(N-1)(N-2) /$ $6 \times N(N-1)(N-2) / 6$ matrix. A configuration $C$ of the system can be labelled by the positions $1 \leq x_{1}<x_{2}<x_{3} \leq N$ of the three particles and if $\psi\left(x_{1}, x_{2}, x_{3}\right)$ is the eigenvector corresponding to the eigenvalue $\lambda$, the equations satisfied by $\psi$ and $\lambda$ are

$$
\lambda \psi\left(x_{1}, x_{2}, x_{3}\right)=\mathrm{e}^{\gamma}\left[\psi\left(x_{1}-1, x_{2}, x_{3}\right)+\psi\left(x_{1}, x_{2}-1, x_{3}\right)+\psi\left(x_{1}, x_{2}, x_{3}-1\right)\right]-3 \psi\left(x_{1}, x_{2}, x_{3}\right) .
$$

These equations are valid only when the positions of the three particles are not neighbours (and $x_{1} \neq 1$ ). If $x_{2}=x_{1}+1$, the hard core condition modifies Eq. (37) and it becomes

$$
\lambda \psi\left(x_{1}, x_{1}+1, x_{3}\right)=\mathrm{e}^{\gamma}\left[\psi\left(x_{1}-1, x_{1}+1, x_{3}\right)+\psi\left(x_{1}, x_{1}+1, x_{3}-1\right)\right]-2 \psi\left(x_{1}, x_{1}+1, x_{3}\right) .
$$

Similarly, when $x_{3}=x_{2}+1$ one obtains

$$
\lambda \psi\left(x_{1}, x_{2}, x_{2}+1\right)=\mathrm{e}^{\gamma}\left[\psi\left(x_{1}-1, x_{2}, x_{2}+1\right)+\psi\left(x_{1}, x_{2}-1, x_{2}+1\right)\right]-2 \psi\left(x_{1}, x_{2}, x_{2}+1\right) .
$$

Lastly, periodic boundary conditions lead to

$$
\lambda \psi\left(1, x_{2}, x_{3}\right)=\mathrm{e}^{\gamma}\left[\psi\left(x_{2}, x_{3}, N\right)+\psi\left(1, x_{2}-1, x_{3}\right)+\psi\left(1, x_{2}, x_{3}-1\right)\right]-3 \psi\left(1, x_{2}, x_{3}\right) .
$$

Usually, solving the Eqs. (37) and (38) for arbitrary $N$ is very hard because the problem is a three-body problem. The idea of the Bethe ansatz consists in trying to see whether a certain simple form for the eigenvector $\psi\left(x_{1}, x_{2}, x_{3}\right)$ can solve the eigenvalue problem. For $P=3$ particles, one looks for an eigenvector which is a sum of 6 terms

$$
\psi\left(x_{1}, x_{2}, x_{3}\right)=A_{123} z_{1}^{x_{1}} z_{2}^{x_{2}} z_{3}^{x_{3}}+A_{213} z_{2}^{x_{1}} z_{1}^{x_{2}} z_{3}^{x_{3}}+\cdots+A_{321} z_{3}^{x_{1}} z_{2}^{x_{2}} z_{1}^{x_{3}}
$$

where the three parameters $z_{1}, z_{2}, z_{3}$ and the 6 parameters $A_{123}, \ldots, A_{321}$ are unknown. Then one inserts this form (41) into Eqs. (37)-(40) to find the conditions that these parameters have to satisfy 
for Eq. (41) to be an eigenvector. If one inserts Eq. (41) into Eq. (37), one finds

$$
\lambda(\gamma)=\mathrm{e}^{\gamma}\left[\frac{1}{z_{1}}+\frac{1}{z_{2}}+\frac{1}{z_{3}}\right]-3
$$

and when one tries to satisfy Eqs. (38) and (39) also, one finds that

$$
\begin{aligned}
& A_{213}=-\frac{\mathrm{e}^{\gamma}-z_{2}}{\mathrm{e}^{\gamma}-z_{1}} A_{123}=-\frac{\mathrm{e}^{\gamma}-z_{1}}{\mathrm{e}^{\gamma}-z_{3}} A_{231}, \\
& A_{312}=-\frac{\mathrm{e}^{\gamma}-z_{3}}{\mathrm{e}^{\gamma}-z_{1}} A_{132}=-\frac{\mathrm{e}^{\gamma}-z_{1}}{\mathrm{e}^{\gamma}-z_{2}} A_{321}, \\
& A_{321}=-\frac{\mathrm{e}^{\gamma}-z_{3}}{\mathrm{e}^{\gamma}-z_{2}} A_{231}=-\frac{\mathrm{e}^{\gamma}-z_{2}}{\mathrm{e}^{\gamma}-z_{1}} A_{312},
\end{aligned}
$$

whereas Eq. (40) gives

$$
\begin{aligned}
& A_{123}=z_{1}^{N} A_{231}=z_{3}^{-N} A_{312}, \\
& A_{231}=z_{2}^{N} A_{312}=z_{1}^{-N} A_{123}, \\
& A_{312}=z_{3}^{N} A_{123}=z_{2}^{-N} A_{231} .
\end{aligned}
$$

One can check that these linear equations for the $A_{i j k}$ are compatible when the $z_{i}$ are solutions of the following three equations:

$$
z_{i}^{-N}\left(\mathrm{e}^{\gamma}-z_{i}\right)^{3}=\prod_{j=1}^{3}\left(\mathrm{e}^{\gamma}-z_{j}\right) .
$$

Therefore, for each choice of the $z_{i}$ which satisfy Eq. (43), expression (41) is an eigenvector with eigenvalue (42). As we want the largest eigenvalue, one has to choose the solution such that $\lambda(\gamma) \rightarrow 0$ as $\gamma \rightarrow 0$. This solution is obtained by choosing the solution of $\left\{z_{1}, z_{2}, z_{3}\right\}$ which converges to $\{1,1,1\}$ as $\gamma \rightarrow 0$ (because the matrix is finite, there is no crossing of eigenvalues, so that the eigenvalue for any $\gamma$ can be obtained by continuity from the solution $\lambda(0)$ for $\gamma=0$ ).

It is straightforward to extend the Bethe ansatz from 3 particles to an arbitrary number $P$ of particles. The eigenvector (41) becomes a sum of $P$ ! terms. The eigenvalue $\lambda(\gamma)$ given by Eqs. (42) and (43) becomes for general $P$ and $N$

$$
\lambda(\gamma)=-p+\mathrm{e}^{\gamma} \sum_{i=1}^{P} \frac{1}{z_{i}},
$$

where the $z_{i}$ satisfy

$$
z_{i}^{-N}\left(\mathrm{e}^{\gamma}-z_{i}\right)^{P}=(-1)^{P-1} \prod_{j=1}^{P}\left(\mathrm{e}^{\gamma}-z_{j}\right) .
$$


Finding the $z_{i}$ solutions of Eq. (45) is not easy. It turns out that the right hand side of Eq. (45) is independent of $i$, thus it can be rewritten as

$$
z_{i}^{-N}\left(z_{i}-\mathrm{e}^{\gamma}\right)^{P}=B
$$

where

$$
B=(-1)^{P-1} \prod_{j=1}^{P}\left(z_{j}-\mathrm{e}^{\gamma}\right) .
$$

Now, we need to select the $P$ roots $z_{i}$ of Eq. (46) which tend to 1 as $\gamma \rightarrow 0$ and $B \rightarrow 0$. To do that we can use the Cauchy formula

$$
\sum_{1 \leq j \leq P} h\left(z_{j}\right)=\oint \frac{\mathrm{d} z}{2 \pi \mathrm{i} z} \frac{\left(z-\mathrm{e}^{\gamma}\right)^{P-1}\left[P z-N\left(z-\mathrm{e}^{\gamma}\right)\right]}{\left(z-\mathrm{e}^{\gamma}\right)^{P}-B z^{N}} h(z),
$$

where the integration contour is a small circle (for small $B$ ) around $\mathrm{e}^{\gamma}$ which surrounds $P$ roots of the polynomial $\left(z-\mathrm{e}^{\gamma}\right)^{P}-B z^{N}$.

Using $h(z)=\left(\mathrm{e}^{\gamma}-z\right) / z$ in Eq. (48), one gets

$$
\lambda(\gamma)=-P \sum_{q=1}^{\infty} \frac{(N q-2) !}{(P q) !(N q-P q-1) !}\left(B \mathrm{e}^{(N-P) \gamma}\right)^{q} .
$$

The $z_{k}$ are solutions of Eq. (46) and depend both on $B$ and $\gamma$ which so far are not related. In principle, one could use these $z_{k}$ into Eq. (47) in order to find a self-consistent equation relating $B$ and $\gamma$. A way of relating $B$ and $\gamma$, simpler than checking that Eq. (47) is satisfied, consists in writing that $z_{1} z_{2} \cdots z_{P}=1$ or equivalently

$$
\sum_{k=1}^{P} \log z_{k}=0
$$

which expresses the fact that the eigenvector associated to the largest eigenvalue is translational invariant $\left(\psi\left(x_{1}, x_{2}, x_{3}\right)=\psi\left(x_{1}+1, x_{2}+1, x_{3}+1\right)\right)$. Using Eq. (48), one can rewrite (50) as

$$
\oint \frac{\mathrm{d} z}{2 \pi \mathrm{i} z} \frac{\left(z-\mathrm{e}^{\gamma}\right)^{P-1}\left[P z-N\left(z-\mathrm{e}^{\gamma}\right)\right]}{\left(z-\mathrm{e}^{\gamma}\right)^{P}-B z^{N}} \log z=0
$$

and by expanding this last expression in powers of $B$, one obtains

$$
\gamma=-\sum_{q=1}^{\infty} \frac{(N q-1) !}{(P q) !(N q-P q) !}\left(B \mathrm{e}^{(N-P) \gamma}\right)^{q} .
$$

The two series (49) and (52) give $\lambda$ as a function of $\gamma$ by eliminating $B$ (this elimination can of course be done for small $B$ but the coefficients of $\lambda$ in powers of $\gamma$ are much more complicated than those appearing in Eqs. (49) and (52); moreover, as the largest eigenvalue $\lambda(\gamma)$ remains isolated as $\gamma$ varies, the function $\lambda(\gamma)$ given by the two series (Eqs. (49) and (52)) for small $\gamma$ can be analytically continued to the whole real axis). 
With the two expressions (49) and (52), it is easy to calculate the successive derivatives of $\lambda$ with respect to $\gamma$. From Eq. (36), one has

$$
v=\lim _{t \rightarrow \infty} \frac{\left\langle Y_{t}\right\rangle}{t}=\left.\frac{1}{P} \frac{\mathrm{d} \lambda}{\mathrm{d} \gamma}\right|_{\gamma=0} \text { and } \Delta=\lim _{t \rightarrow \infty} \frac{\left\langle Y_{t}^{2}\right\rangle-\left\langle Y_{t}\right\rangle^{2}}{t}=\left.\frac{1}{2 P^{2}} \frac{\mathrm{d}^{2} \lambda}{\mathrm{d} \gamma^{2}}\right|_{\gamma=0}
$$

and this leads to Eq. (9). In fact, $\lambda(\gamma)$ contains also all the higher cumulants of $Y_{t}$ and it can be used to calculate the large deviation function [28].

In the case of open boundary conditions [24] (Section 3) as well for a ring with a partial asymmetry [29], the diffusion constant has been calculated by an extension of the matrix method of Section 3. The calculation by the Bethe ansatz has not been extended yet to these cases.

\section{Two species of particle on a ring}

The matrix method of Section 3 can be generalized to obtain the exact steady state of systems of more complicated systems, in particular, systems with two species of particles [30]. Consider a ring of $N$ sites with two species of particles represented by 1 and 2, and holes represented by 0 and in which the hopping rates are

$$
\begin{aligned}
& 10 \rightarrow 01 \text { with rate } 1, \\
& 20 \rightarrow 02 \text { with rate } \alpha, \\
& 12 \rightarrow 21 \text { with rate } \beta .
\end{aligned}
$$

One can show [30] that the steady state weights may be written as

$$
\operatorname{trace}\left(X_{1} X_{2} \cdots X_{N}\right)
$$

where $X_{i}=D$ if site $i$ is occupied by a 1 particle, $X_{i}=A$ if it is occupied by a 2 particle and $X_{i}=E$ if it is empty, provided that the matrices $D, A$ and $E$ satisfy the following algebra:

$$
D E=D+E ; \quad \beta D A=A ; \quad \alpha A E=A .
$$

The last two of these equations are satisfied when $A$ is given by

$$
A=|V\rangle\langle W|,
$$

and

$$
D|V\rangle=\frac{1}{\beta}|V\rangle ; \quad\langle W| E=\frac{1}{\alpha}\langle W| .
$$

So, one can use the same matrices $D, E$ as those of Section 2 for the case of open boundary conditions, and construct matrix $A$ from the vectors $\langle W|$ and $|V\rangle$.

The proof that Eqs. (55) and (56) gives the steady state of the two species problem is very similar to the proof for the case of open boundary conditions: one shows that the loss term and the gain 
term for the probability of each configuration are equal. For example, for a configuration of the type

$$
\underbrace{00 \cdots 0}_{p} \underbrace{1 \cdots 1}_{q} \underbrace{2 \underbrace{2 \cdots 2}}_{r} \underbrace{0 \cdots 0}_{s}
$$

on a ring, the loss term during $\mathrm{d} t$ is

$$
(\alpha+\beta) \operatorname{trace}\left(E^{p} D^{q} A^{r} E^{s}\right) \mathrm{d} t
$$

as there are only two possibilities of leaving this configuration and the gain term is

$$
\operatorname{trace}\left(E^{p-1} D E D^{q-1} A^{r} E^{s}\right) \mathrm{d} t
$$

as there is a single way of entering this configuration. For such configurations, it is almost evident from Eq. (56) that the loss term and the gain term coincide.

To illustrate a situation with two species, let us consider on a ring of $N$ sites a single particle 2 and $P$ particles 1 [31,32]. The parameters $\alpha$ and $\beta$ which appear in Eq. (54) are chosen such that

$$
\beta<1-\alpha .
$$

This implies in particular that $\alpha<1$ and $\beta<1$, so that the particle 2 is slower than particles 1 and as $\beta<1$, it plays the role of a moving obstacle. In other words, particles 1 can be thought as cars and the particle 2 as a single slow truck.

Using the algebraic rules (56), it is possible to calculate $Y(N, P)$ defined by

$$
Y(N, P)=\frac{1}{\langle W \mid V\rangle_{\tau_{1}=0,1}} \sum_{\tau_{N-1}=0,1} \delta_{\tau_{1}+\cdots+\tau_{N-1}, P}\left\langle W\left|\prod_{i=1}^{N-1}\left[\tau_{i} D+\left(1-\tau_{i}\right) E\right]\right| V\right\rangle
$$

and show that for $1 \leq P \leq N-2$

$$
Y(N, P)=\sum_{p=0}^{N-P-1} \sum_{q=0}^{P} \frac{1}{\alpha^{p} \beta^{q}} \frac{(N-2-p) !(N-2-q) ![p(P-q)+q(N-1-P)]}{P !(N-1-P) !(P-q) !(N-1-P-p) !},
$$

whereas $Y(N, 0)=\alpha^{-(N-1)}$ and $Y(N, N-1)=\beta^{-(N-1)}$. Then the average velocity $v_{2}$ of particle 2 (the truck) is given by

$$
v_{2}=\frac{Y(N-1, P)-Y(N-1, P-1)}{Y(N, P)},
$$

whereas the average velocity $v_{1}$ of particles 1 (cars) is given by

$$
v_{1}=v_{2}+\frac{N}{P} \frac{Y(N-1, P-1)}{Y(N, P)} .
$$

These velocities are shown in Fig. 3 as a function of the density $\rho=P / N$ of particles 1 for two system sizes $(N=100$ and $N=200)$. For large $N$, we see that as $\rho$ varies, $v_{2}$ presents a plateau for $1-\alpha<\rho<\beta$ where $v_{2}=\alpha-\beta$. Along this plateau, the ring consists of two macroscopic regions: a region of high density $\rho_{+}=1-\alpha$ following the truck and a region of low density $\beta$ 


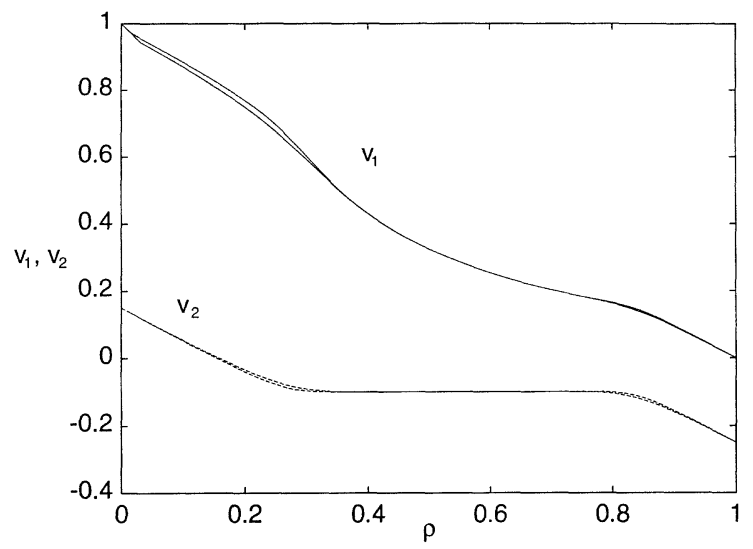

Fig. 3. The velocities $v_{1}$ and $v_{2}$ of the particles 1 and 2 versus the density $\rho$ of first class particles for two rings of 100 and 200 sites when $\alpha=0.15$ and $\beta=0.25$. Finite size effects are small enough to make the curves for 100 and 200 hard to distinguish.

ahead of the truck. These two regions are of length $L_{+}=N(\rho-\beta) /(1-\alpha-\beta)$ and $L_{-}=$ $N(1-\alpha-\rho) /(1-\alpha-\beta)$ so that the total density is

$$
\rho=\frac{L_{+} \rho_{+}+L_{-} \rho_{-}}{N} .
$$

The expression of the average velocity $v_{1}$ of the particles 1 can also be obtained in this coexistence region, by

$$
v_{1}=\frac{L_{+} \rho_{+} v_{+}+L_{-} \rho_{-} v_{-}}{P}
$$

with $v_{ \pm}=1-\rho_{ \pm}$i.e.

$$
v_{1}=\alpha-\beta+\frac{(1-\alpha) \beta}{\rho} .
$$

In the thermodynamic limit, three phases can be observed as the density $\rho=P / N$ of particles 1 varies. The steady state velocities $v_{1}$ of the particles 1 and $v_{2}$ of the particle 2 have the following expressions (which can be obtained from the asymptotic behavior of Eq. (63)):

For $\rho<\beta, \quad v_{1}=1-\rho$;

For $\beta<\rho<1-\alpha, \quad v_{1}=\alpha-\beta+(1-\alpha) \beta / \rho ; \quad v_{2}=\alpha-\beta$.

For $1-\alpha<\rho, \quad v_{1}=1-\rho$;

$$
\begin{aligned}
& v_{2}=\alpha-\rho . \\
& v_{2}=\alpha-\beta . \\
& v_{2}=1-\beta-\rho .
\end{aligned}
$$

It is interesting to notice that in the range $\beta<\rho<1-\alpha$, the truck (particle 2) has a constant velocity, whereas in that range, cars (particles 1) have an average velocity slower than $1-\rho$ (the velocity in absence of the truck). In this whole range there is a coexistence of two macroscopic 
regions, one of low density $\beta$ and one of high density $1-\alpha$, very similar to the coexistence between the liquid and the gas for a fluid.

A case of the two species problem of particular interest and which, as we will see in Section 6, can be used to study shocks is that of first and second class particles [33-36]. This corresponds to $\alpha=\beta=1$ in Eq. (54) so that all hopping rates are 1 . Both first and second class particles hop forward when they have a hole to their right, but when a first class particle has a second class particle to its right, the two particles interchange positions. Thus, a second class particle tends to move backwards in an environment of a high density of first class particles and tends to move forwards in an environment of a low density of first class particles so that it gets trapped by a shock. The matrix method was used in [30] to calculate exactly the profile of a shock as seen from a second class particle by starting from the steady state weights (55) of the two species problem on a ring. We are going to see in Section 6 that the shock profile can also be calculated directly on an infinite line.

\section{Shock profile}

It is possible to generalize the calculation of Sections 3 and 5 to describe shock profiles in an infinite system.

Consider an infinite system with only one kind of particles (as in Sections 2-4). If the initial condition is a Bernoulli measure with density $\rho_{+}$at the right of the origin (i.e. the $\tau_{i}$ are independent and $\left\langle\tau_{i}\right\rangle=\rho_{+}$for $\left.i \geq 0\right)$ and with density $\rho_{-}$at the left of the origin $\left(\left\langle\tau_{i}\right\rangle=\rho_{-}\right.$for $\left.i<0\right)$, the evolution of the ASEP develops a shock. The shock has a velocity

$$
v=1-\rho_{-}-\rho_{+}
$$

and this can be easily understood from the conservation of mass. The current far to the right of the shock is $\rho_{+}\left(1-\rho_{+}\right)$and far to the left is $\rho_{-}\left(1-\rho_{-}\right)$. Therefore, in a large region containing the shock, the number of particles increases by $\left[\rho_{-}\left(1-\rho_{-}\right)-\rho_{+}\left(1-\rho_{+}\right)\right] t$ during the time $t$. This increase in the number of particles has the effect of a translation of the shock of $v t$ so that

$$
\left(\rho_{-}-\rho_{+}\right) v t=\left[\rho_{-}\left(1-\rho_{-}\right)-\rho_{+}\left(1-\rho_{+}\right)\right] t
$$

which gives (66).

A more delicate question is that of the shape of a shock. Because the dynamics is stochastic, one first needs to locate the shock. There exist several ways of locating the shock. For example, one can choose [38] an irrational value $\rho_{*}$ which satisfies $\rho_{-}<\rho_{*}<\rho_{+}$and define the position of the shock as the value $m_{*}$ which minimizes $S_{m}$

$$
S_{m}=\left\{\begin{array}{cl}
\sum_{k=1}^{m}\left(\tau_{k}-\rho_{*}\right), & \text { for } m>0, \\
0, & \text { for } m=0, \\
-\sum_{k=-m+1}^{0}\left(\tau_{k}-\rho_{*}\right), & \text { for } m<0,
\end{array}\right.
$$

(as the density is $\rho_{+}$to the right of the shock, the sum increases like $\left(\rho_{ \pm}-\rho_{*}\right) m$ as $m \rightarrow \pm \infty$, thus, for almost all configurations $\left\{\tau_{n}\right\}$, there is a unique minimum $m_{*}$ ). With this definition of the 
position of the shock (which depends on $\rho_{*}$ ), one can study the measure seen from this position. For example, the profile $\left\langle\tau_{n}\right\rangle$ is just the average occupation at position $m_{*}+n$ (given that the minimum of Eq. (67) is located at $m_{*}$ ). Obviously, as the location of the minimum depends on the value of $\rho_{*}$, the profile $\left\langle\tau_{n}\right\rangle$ or the measure seen from the minimum $m_{*}$ will depend on $\rho_{*}$. This means that two observers looking at exactly the same physical situation, but using two different values of $\rho_{*}$ to locate the shock, will observe different profiles. The profile $\left\langle\tau_{n}\right\rangle$ is, therefore, not an intrinsic property of the shock.

One can, however, define some properties of shocks [38] like sums of the type

$$
\Phi_{k}=\sum_{n=-\infty}^{\infty}\left\langle\left(\tau_{n}-\rho_{-}\right)\left(\tau_{n+k}-\rho_{+}\right)\right\rangle,
$$

which are independent of the definition chosen to locate the shock and can therefore be thought as intrinsic properties of the shock. For example [38], one expects $\Phi_{1}$ to be always given by

$$
\Phi_{1}=\sum_{n=-\infty}^{\infty}\left\langle\left(\tau_{n}-\rho_{-}\right)\left(\tau_{n+1}-\rho_{+}\right)\right\rangle=\rho_{-}\left(1-\rho_{+}\right)
$$

irrespective of the method chosen to locate the shock, in particular irrespective of the value of $\rho_{*}$.

A convenient way of locating the shock consists in replacing one hole in the system by a second class particle (i.e. with dynamics given by Eq. (54) with $\alpha=\beta=1$ ). This replacement does not affect the dynamics of the particles 1 and so does not disturb the configurations of the shock. The second class particle has a larger velocity in the region of density $\rho_{-}$than in the region of density $\rho_{+}$and one can show that it gets trapped by the shock [30,33-35,37]. Therefore, the position of the second class particle can be used to locate the shock.

One can try to describe the measure seen from this second class particle. It is possible to show $[22,37,38]$ that the steady state weights of all possible environments of the second class particle in presence of a shock characterized by the two asymptotic densities $\rho_{+}$and $\rho_{-}$can be written in a matrix form similar to Eq. (16).

If the occupation numbers $\tau_{i}$ are specified for the $n$ sites at the left of second class particle and the $n^{\prime}$ sites at its right, the steady state probability of the configuration is given by

$$
\operatorname{Prob}\left(\tau_{-n} \cdots \tau_{-1} ; \tau_{1} \cdots \tau_{n^{\prime}}\right)=\left\langle W\left|\left[\prod_{i=-n}^{-1}\left(D \tau_{i}+E\left(1-\tau_{i}\right)\right)\right] A\left[\prod_{i=1}^{n^{\prime}}\left(D \tau_{i}+E\left(1-\tau_{i}\right)\right)\right]\right| V\right\rangle
$$

when the matrices $A, D, E$ and the vectors $|V\rangle$ and $\langle W|$ satisfy

$$
\begin{aligned}
& D E=\left(1-\rho_{-}\right)\left(1-\rho_{+}\right) D+\rho_{-} \rho_{+} E, \\
& D A=\rho_{-} \rho_{+} A ; \quad A E=\left(1-\rho_{-}\right)\left(1-\rho_{+}\right) A, \\
& \langle W|(D+E)=\langle W|; \quad(D+E)| V\rangle=| V\rangle, \\
& \langle W|A| V\rangle=1 .
\end{aligned}
$$

For example, Prob(1 1102211101$)=\left\langle W\left|D^{2} E A D^{2} E D\right| V\right\rangle$.

So as in Section 5, for each particle we put a $D$, for each hole an $E$ and for the second class particle we put the matrix $A$. As in Section 3, it is easy to see that all the matrix elements of the form 
$\left\langle W\left|D^{m} A E^{n}\right| V\right\rangle$ can be calculated immediately from Eq. (71)

$$
\left\langle W\left|D^{n} A E^{n^{\prime}}\right| V\right\rangle=\left[\rho_{-} \rho_{+}\right]^{n}\left[\left(1-\rho_{-}\right)\left(1-\rho_{+}\right)\right]^{n^{\prime}}
$$

and that, by using the algebra (71), all the other matrix elements can be reduced by using the algebra to such elements.

One can calculate that way the profile seen from the second class particle

$$
\left\langle\tau_{n}\right\rangle= \begin{cases}\left\langle W\left|A(D+E)^{n-1} D\right| V\right\rangle & \text { for } n \geq 1 \\ \left\langle W\left|D(D+E)^{-n+1} A\right| V\right\rangle & \text { for } n \leq-1 .\end{cases}
$$

One finds in particular

$$
\left\langle\tau_{1}\right\rangle=\rho_{+}+\rho_{-}-\rho_{+} \rho_{-} ; \quad\left\langle\tau_{-1}\right\rangle=\rho_{+} \rho_{-} ; \quad\left\langle\tau_{-2}\right\rangle=\rho_{+} \rho_{-}+\rho_{-} \rho_{+}\left(1-\rho_{-}\right)\left(1-\rho_{+}\right)
$$

and so on, in agreement with [30]. One can check that the velocity $v$ of the second class particle given by

$$
v=\left\langle\left(1-\tau_{1}\right)-\tau_{-1}\right\rangle=1-\rho_{+}-\rho_{-}
$$

coincides with the shock velocity (66), as it should since the second class particle is trapped by the shock.

The algebra (71) which gives the measure seen from the second class particle, can of course be used to calculate all the intrinsic properties of the shock, in particular the sums $\Phi_{k}$ defined in Eq. (68). It is possible to show [38] from the algebra (71) that there is a relation between the profile $\left\langle\tau_{n}\right\rangle$ seen from the second class particle and the intrinsic sums (68)

$$
\Phi_{k+1}-\Phi_{k}=\left(\rho_{+}-\rho_{-}\right)\left(\rho_{+}-\left\langle\tau_{k}\right\rangle\right) .
$$

Of course such a relation has no reason to remain valid when the profile $\left\langle\tau_{k}\right\rangle$ is seen from a different location than the second class particle.

\section{Conclusions}

The matrix method described in Sections 3, 5 and 6 has been extended recently to a number of cases: parallel dynamics [39,40], partial asymmetry [17,37,41-44], transient properties [45], systems with two or more species of particles [46-49], reaction diffusion models [50,51], cases with disorder [52]. However, there remains a number of simple generalizations of the asymmetric exclusion model such as the case of a fixed blockage [53], the calculation of general unequal time correlations [54], more general reaction diffusion models [55], open systems with several species of particles [56], for which exact solutions are still lacking.

Trying to extend the Bethe ansatz calculation of Section 4 to other cases (several species, partial asymmetry, open boundary conditions) would certainly lead to new interesting results and make more transparent the link with the matrix method. 


\section{Acknowledgements}

The content of these lectures is based on work done in collaboration with E. Domany, M.R. Evans, S. Goldstein, V. Hakim, S. Janowsky, J.L. Lebowitz, K. Mallick, D. Mukamel, V. Pasquier, E.R. Speer. I would like to thank them as well as D. Kim, V. Rittenberg, G. Schütz, H. Spohn and R. Stinchcombe for useful discussions.

\section{References}

[1] F. Spitzer, Adv. Math. 5 (1970) 246.

[2] T.M. Liggett, Interacting Particle Systems, Springer, New York, 1985.

[3] H. Spohn, Large Scale Dynamics of Interacting Particles, Springer, New York, 1991.

[4] J. Krug, Phys. Rev. Lett. 67 (1991) 1882.

[5] B. Schmittmann, R.K.P. Zia, Statistical mechanics of driven diffusive systems, in: C. Domb, J.L. Lebowitz (Eds.), Phase Transitions and Critical Phenomena, vol. 17, Academic, New York, 1995.

[6] J.L. Lebowitz, E. Presutti, H. Spohn, J. Statist. Phys. 51 (1988) 841.

[7] T. Halpin-Healy, Y.-C. Zhang, Phys. Rep. 254 (1994) 215.

[8] A. De Masi, E. Presutti, E. Scacciatelli, Ann. Inst. Henri Poincaré: Probabilités et Statistiques 25 (1989) 1.

[9] C. Kipnis, S. Olla, S.R.S. Varadhan, Commun. Pure Appl. Math. 42 (1989) 115.

[10] J.L. Lebowitz, E. Orlandi, E. Presutti, Physica D 33 (1988) 165.

[11] P. Meakin, P. Ramanlal, L.M. Sander, R.C. Ball, Phys. Rev. A 34 (1986) 5091.

[12] A. De Masi, P.A. Ferrari, J. Statist. Phys. 38 (1985) 603.

[13] R. Kutner, H. van Beijeren, J. Statist. Phys. 39 (1985) 317.

[14] S.N. Majumdar, M. Barma, Phys. Rev. B 44 (1991) 5306.

[15] B. Derrida, M.R. Evans, D. Mukamel, J. Phys. A 26 (1993) 4911.

[16] B. Derrida, E. Domany, D. Mukamel, J. Stat. Phys. 69 (1992) 667.

[17] B. Derrida, M.R. Evans, V. Hakim, V. Pasquier, J. Phys. A 26 (1993) 1493.

[18] G. Schütz, E. Domany, J. Stat. Phys. 72 (1993) 277.

[19] L.D. Faddeev, Sov. Sci. Rev. C 1 (1980) 107.

[20] R.J. Baxter, Exactly Solved Models in Statistical Mechanics, Academic, New York, 1982.

[21] V. Hakim, J.P. Nadal, J. Phys. A 16 (1983) L213.

[22] B. Derrida, M.R. Evans, in: V. Privman (Ed.), Non-equilibrium Statistical Mechanics in One Dimension, Cambridge University Press, Cambridge, 1997, pp. 277.

[23] P. Ruján, J. Stat. Phys. 49 (1987) 139.

[24] B. Derrida, M.R. Evans, K. Mallick, J. Stat. Phys. 79 (1995) 833.

[25] D. Dhar, Phase Transitions 9 (1987) 51.

[26] L.H. Gwa, H. Spohn, Phys. Rev. A 46 (1992) 844.

[27] D. Kim, Phys. Rev. E 52 (1995) 3512; J. Phys. A 30 (1997) 3817.

[28] B. Derrida, J.L. Lebowitz, Phys. Rev. Lett. 80 (1998) 209.

[29] B. Derrida, K. Mallick, J. Phys. A 30 (1997) 1031.

[30] B. Derrida, S.A. Janowsky, J.L. Lebowitz, E.R. Speer, Europhys. Lett. 22 (1993) 651; J. Stat. Phys. 73 (1993) 813.

[31] B. Derrida, in: Hao Bailin (Ed.), Stat. Phys. 19, Xiamen, China, World Scientific, Singapore, 1996, pp. $243-253$.

[32] K. Mallick, J. Phys. A 29 (1996) 5375.

[33] E.D. Andjel, M. Bramson, T.M. Liggett, Probab. Theory Rel. Fields 78 (1988) 231.

[34] P.A. Ferrari, C. Kipnis, E. Saada, Ann. Probab. 19 (1991) 226.

[35] C. Boldrighini, G. Cosimi, S. Frigio, M.G. Nuñes, J. Stat. Phys. 55 (1989) 611.

[36] E.R. Speer, The two species totally asymmetric exclusion process, in: M. Fannes, C. Maes, A. Verbeure (Eds.), Micro, Meso and Macroscopic Approaches in Physics, Plenum Press, New York, 1994.

[37] B. Derrida, J.L. Lebowitz, E.R. Speer, J. Stat. Phys. 89 (1997) 135. 
[38] B. Derrida, S. Goldstein, J.L. Lebowitz, E.R. Speer, J. Stat. Phys., in press.

[39] H. Hinrichsen, J. Phys. A 29 (1996) 3659.

[40] A. Honecker, I. Peschel, J. Stat. Phys. 88 (1997) 319.

[41] S. Sandow, Phys. Rev. 50 (1994) 2660.

[42] S. Sandow, G.M. Schütz, Europhys. Lett. 26 (1994) 7.

[43] F.H.L. Essler, V. Rittenberg, J. Phys. A 29 (1996) 3375.

[44] K. Mallick, S. Sandow, J. Phys. A 30 (1997) 4513.

[45] R.B. Stinchcombe, G.M. Schütz, Europhys. Lett. 29 (1995) 663; Phys. Rev. Lett. 75 (1995) 140.

[46] M.R. Evans, Y. Kafri, M.H. Koduvely, D. Mukamel, Phys. Rev. Lett. 80 (1998) 425.

[47] P.F. Arndt, T. Heinzel, V. Rittenberg, J. Phys. A 31 (1998) 833; J. Stat. Phys. 90 (1998) 783.

[48] F.C. Alcaraz, S. Dasmahapatra, V. Rittenberg, J. Phys. A 31 (1998) 845.

[49] A.B. Kolomeisky, preprint, 1997.

[50] H. Hinrichsen, S. Sandow, I. Peschel, J. Phys. A 29 (1996) 2643.

[51] M.J.E. Richardson, M.R. Evans, J. Phys. A 30 (1997) 811.

[52] M.R. Evans, Europhys. Lett. 36 (1996) 13; J. Phys. A 30 (1997) 5669.

[53] S.A. Janowsky, J.L. Lebowitz, Phys. Rev. A 45 (1992) 618.

[54] G.M. Schütz, J. Stat. Phys. 88 (1997) 427.

[55] F.C. Alcaraz, M. Droz, M. Henkel, V. Rittenberg, Ann. Phys. (N.Y.) 230 (1994) 250.

[56] M.R. Evans, D.P. Foster, C. Godrèche, D. Mukamel, Phys. Rev. Lett. 74 (1995) 208. 